\title{
Which Is Better to Prevent Cardiovascular Events, Only EPA or the Combination of EPA and DHA?
}

\author{
Hidekatsu Yanai
}

Anti-atherosclerotic effects of n-3 fatty acids (FA) include reduction of serum triglyceride (TG), increase of serum highdensity lipoprotein (HDL), anti-platelets effects, anti-inflammatory effects, improvement of endothelial function, increased stability of plaque and improvement of blood rheology [1].

Very recently, we studied the effects of the switching from high-purity eicosapentaenoic acid ester (hp-EPA) to n-3 FA formulation containing EPA and docosahexaenoic acid (DHA) on metabolic parameters [2]. In patients with baseline $\mathrm{TG} \geq$ $150 \mathrm{mg} / \mathrm{dL}$, TG tended to decrease. In the analysis of patients who underwent the switching to the formulation including the same daily dose of EPA and DHA, in patients with baseline $\mathrm{TG} \geq 150 \mathrm{mg} / \mathrm{dL}$, TG significantly decreased, indicating that the switching is more effective to reduce TG in patients with higher TG levels at baseline.

In the epidemiological study, the multivariate relative risk of coronary heart disease adjusting for coronary risk factors and time since last meal associated with a $1-\mathrm{mmol} / \mathrm{L}$ increase in TG was 1.29 (95\% confidence interval (CI): $1.09-1.53 ; \mathrm{P}=$ $0.004)$ for men and $1.42(95 \% \mathrm{CI}: 1.15-1.75 ; \mathrm{P}=0.001)$ for women, suggesting that elevation of serum TG is a crucial risk factor for cardiovascular events [3]. Considering that, together with our result, the combination of EPA and DHA is more effective to prevent cardiovascular events than only EPA.

Statins are the "gold standard" for the treatment to prevent cardiovascular diseases. To evaluate the cardio-protective effects of some drugs in clinical trials, we should consider whether trial participants take statins or not. In Japan EPA lipid intervention study (JELIS), all participants had taken statins (Table 1) [4-8]. The primary endpoint was detected in 262 (2.8\%) patients in the EPA group and $324(3.5 \%)$ in controls, a $19 \%$ relative reduction in major coronary events $(\mathrm{P}=0.011)$.

In the GISSI-Prevenzione (GISSI-P) trial, the treatment with n-3 FA (EPA + DHA) significantly lowered the risk of the primary endpoint (relative risk decrease 15\% (95\% CI, 2 - 26)) [5]. In the GISSI-HF trial, 955 (27\%) patients died from any cause in the n-3 PUFA (EPA + DHA) group and 1,014 (29\%) in the placebo group (adjusted hazard ratio (HR) 0.91 (95.5\%

Manuscript accepted for publication July 27, 2016

Department of Internal Medicine, National Center for Global Health and Medicine Kohnodai Hospital, 1-7-1 Kohnodai, Ichikawa, Chiba 272-0034, Japan. Email: dyanai@hospk.ncgm.go.jp

doi: http://dx.doi.org/10.14740/jem359w
CI: $0.833-0.998), P=0.041)$ [6]. One thousand nine hundred eighty-one (57\%) patients in the n-3 FA group and 2,053 $(59 \%)$ in the placebo group died or were admitted to hospital for cardiovascular reasons (adjusted HR 0.92 (99\% CI: 0.849 $0.999), \mathrm{P}=0.009)$. The percentages of subjects who had taken satins were only $4.7 \%$ and $22.6 \%$ in GISSI-P and GISSI-HF trials, respectively.

In the recent OMEGA study, the event rates were (n-3 FA (EPA + DHA) and control groups) as follows: sudden cardiac death, $1.5 \%$ and $1.5 \%(\mathrm{P}=0.84)$; total mortality, $4.6 \%$ and $3.7 \%(\mathrm{P}=0.18)$; major adverse cerebrovascular and cardiovascular events, $10.4 \%$ and $8.8 \%(\mathrm{P}=0.1)$; and revascularization in survivors, $27.6 \%$ and $29.1 \%(\mathrm{P}=0.34)$ [7]. In the ORIGIN trial, the incidence of the primary outcome was not significantly decreased among patients receiving n-3 FA (EPA + DHA), as compared with those receiving placebo (574 patients $(9.1 \%)$ vs. 581 patients $(9.3 \%)$; hazard ratio: $0.98 ; 95 \%$ CI: 0.87 - 1.10; $\mathrm{P}=0.72)$ [8].

In the previous two studies (GISSI-P and GISSI-HF), EPA + DHA may be effective to prevent cardiovascular events because of fewer combined use of statins. In the recent studies (OMEGA and ORIGIN) which included many participants who had taken statins, EPA + DHA failed to reduce cardiovascular events. However, EPA demonstrated a significant reduction of cardiovascular events in JELIS, in which all participants had taken statins.

We should consider how EPA or DHA reduces cardiovascular events independently of TG reduction. EPA or DHA may reduce cardiovascular events, due to decrease of inflammation and/or platelets activity, or the improvement of vascular integrity. Significant but slight differences in TG reduction between the n-3 FA group and controls in JELIS, GISSI-P and GISSIHF support my hypothesis.

\section{Conflicts of Interest}

The author declares no conflicts of interest concerning this article.

\section{References}

1. Yanai H, Yoshida H. Eicosapentaenoic Acids (EPA) for Atherosclerotic Diseases. J JSMUFF. 2014;8(3):215-220.

2. Katsuyama H, Matsuura S, Yanai H. Effects of the 


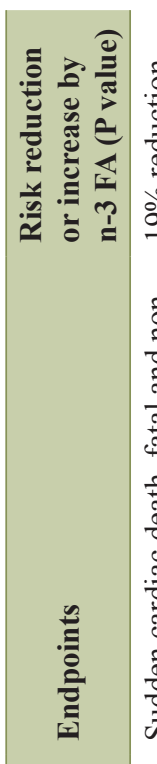

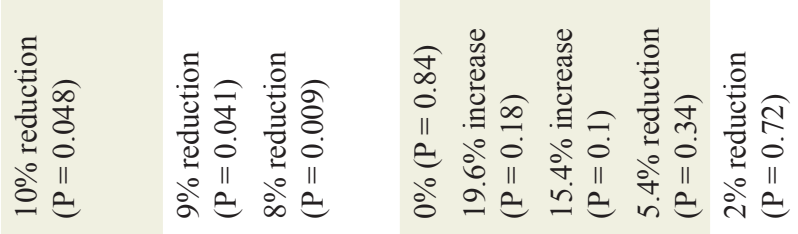

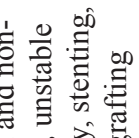

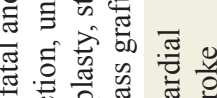

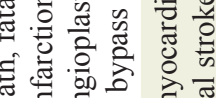

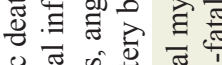

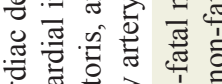
ठี

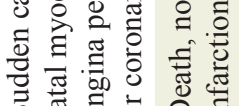

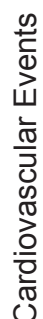

$\stackrel{8}{*}$

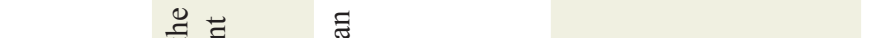

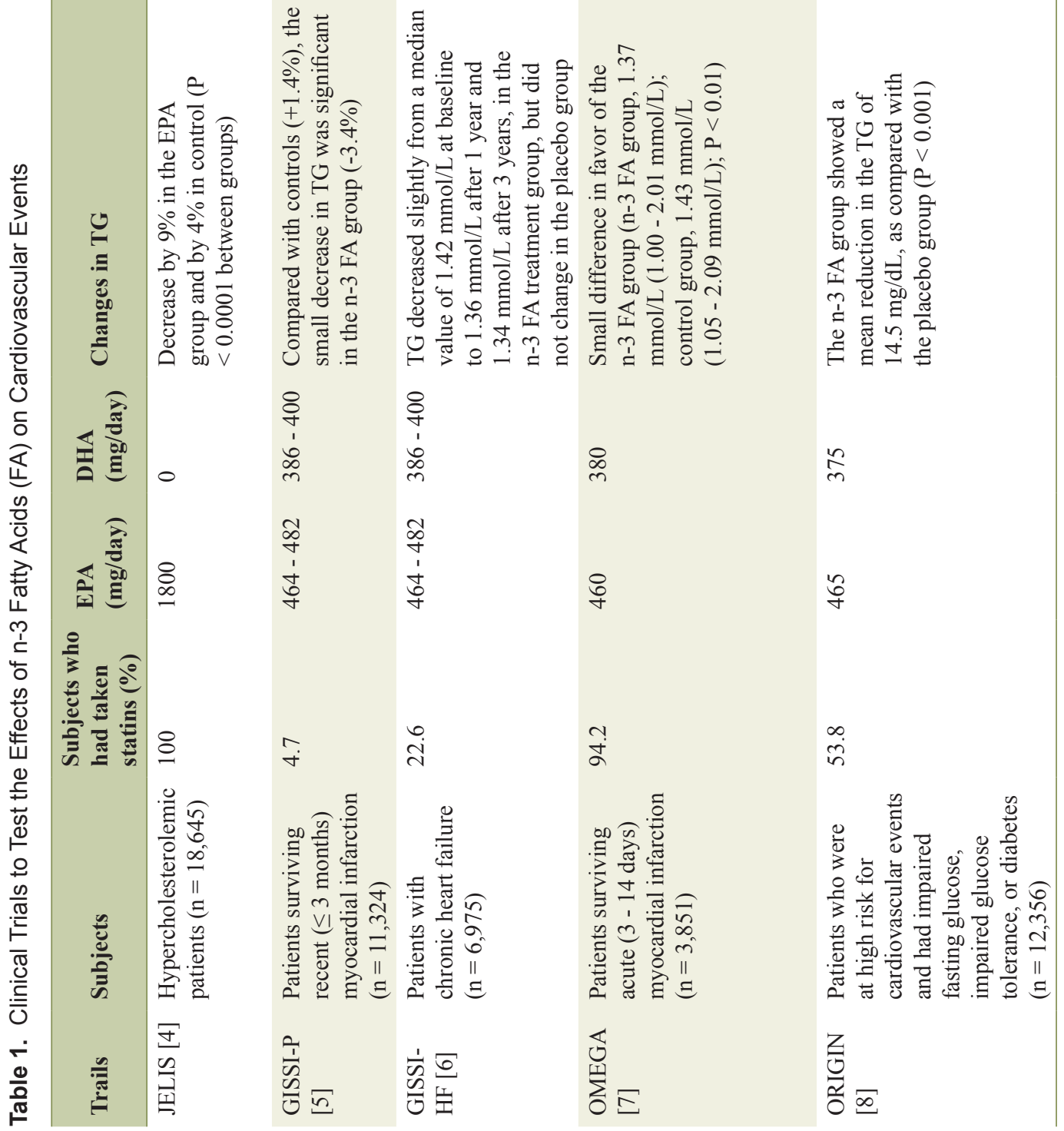


Switching from High-Purity Eicosapentaenoic Acid to Combination of Eicosapentaenoic Acid and Docosahexaenoic Acid on Metabolic Parameters: A Retrospective Longitudinal Study. J Endocrinol Metab. 2016;6(3):7579.

3. Iso H, Naito Y, Sato S, Kitamura A, Okamura T, Sankai T, Shimamoto T, et al. Serum triglycerides and risk of coronary heart disease among Japanese men and women. Am J Epidemiol. 2001;153(5):490-499.

4. Yokoyama M, Origasa H, Matsuzaki M, Matsuzawa Y, Saito Y, Ishikawa Y, Oikawa S, et al. Effects of eicosapentaenoic acid on major coronary events in hypercholesterolaemic patients (JELIS): a randomised open-label, blinded endpoint analysis. Lancet. 2007;369(9567):10901098.

5. Dietary supplementation with n-3 polyunsaturated fatty acids and vitamin $\mathrm{E}$ after myocardial infarction: results of the GISSI-Prevenzione trial. Gruppo Italiano per lo Stu- dio della Sopravvivenza nell'Infarto miocardico. Lancet. 1999;354(9177):447-455.

6. Tavazzi L, Maggioni AP, Marchioli R, Barlera S, Franzosi MG, Latini R, Lucci D, et al. Effect of n-3 polyunsaturated fatty acids in patients with chronic heart failure (the GISSI-HF trial): a randomised, double-blind, placebo-controlled trial. Lancet. 2008;372(9645):12231230 .

7. Rauch B, Schiele R, Schneider S, Diller F, Victor N, Gohlke H, Gottwik M, et al. OMEGA, a randomized, placebo-controlled trial to test the effect of highly purified omega- 3 fatty acids on top of modern guidelineadjusted therapy after myocardial infarction. Circulation. 2010;122(21):2152-2159.

8. Bosch J, Gerstein HC, Dagenais GR, Diaz R, Dyal L, Jung H, Maggiono AP, et al. n-3 fatty acids and cardiovascular outcomes in patients with dysglycemia. N Engl J Med. 2012;367(4):309-318. 